\title{
Co-infection in critically ill patients with COVID-19: an observational cohort study from England
}

\author{
Vadsala Baskaran 1,2,3,*, Hannah Lawrence ${ }^{1,2,3}$, Louise E. Lansbury², Karmel Webb², Shahideh Safavi ${ }^{3,4}$, Nurul I. Zainuddin', \\ Tausif Huq ${ }^{1}$, Charlotte Eggleston ${ }^{1}$, Jayne Ellis ${ }^{5}$, Clare Thakker ${ }^{5}$, Bethan Charles ${ }^{6}$, Sara Boyd ${ }^{7.8}$, Tom Williams 7 , \\ Claire Phillips 9 , Ethan Redmore ${ }^{9}$, Sarah Platt ${ }^{10}$, Eve Hamilton ${ }^{10}$, Andrew Barr ${ }^{10}$, Lucy Venyo ${ }^{10}$, Peter Wilson ${ }^{5}$, \\ Tom Bewick ${ }^{11}$, Priya Daniel ${ }^{11}$, Paul Dark ${ }^{6,12}$, Adam R. Jeans ${ }^{6}$, Jamie McCanny ${ }^{7}$, Jonathan D. Edgeworth ${ }^{7}$, Martin \\ J. Llewelyn ${ }^{9}$, Matthias L. Schmid ${ }^{10}$, Tricia M. McKeever ${ }^{2,3}$, Martin Beed ${ }^{13,14}$ and Wei Shen Lim ${ }^{1,3}$
}

\begin{abstract}
Introduction. During previous viral pandemics, reported co-infection rates and implicated pathogens have varied. In the 1918 influenza pandemic, a large proportion of severe illness and death was complicated by bacterial co-infection, predominantly Streptococcus pneumoniae and Staphylococcus aureus.
\end{abstract}

Gap statement. A better understanding of the incidence of co-infection in patients with COVID-19 infection and the pathogens involved is necessary for effective antimicrobial stewardship.

Aim. To describe the incidence and nature of co-infection in critically ill adults with COVID-19 infection in England.

Methodology. A retrospective cohort study of adults with COVID-19 admitted to seven intensive care units (ICUs) in England up to 18 May 2020, was performed. Patients with completed ICU stays were included. The proportion and type of organisms were determined at $<48$ and $>48 \mathrm{~h}$ following hospital admission, corresponding to community and hospital-acquired co-infections.

Results. Of 254 patients studied (median age 59 years (IQR 49-69); 64.6\% male), 139 clinically significant organisms were identified from $83(32.7 \%)$ patients. Bacterial co-infections/ co-colonisation were identified within $48 \mathrm{~h}$ of admission in 14 (5.5\%) patients; the commonest pathogens were Staphylococcus aureus (four patients) and Streptococcus pneumoniae (two patients). The proportion of pathogens detected increased with duration of ICU stay, consisting largely of Gram-negative bacteria, particularly Klebsiella pneumoniae and Escherichia coli. The co-infection/ co-colonisation rate $>48 \mathrm{~h}$ after admission was 27/1000 person-days $(95 \% \mathrm{Cl} 21.3-34.1)$. Patients with co-infections/ co-colonisation were more likely to die in ICU (crude OR $1.78,95 \% \mathrm{Cl} 1.03-3.08, P=0.04)$ compared to those without co-infections/ co-colonisation.

Conclusion. We found limited evidence for community-acquired bacterial co-infection in hospitalised adults with COVID-19, but a high rate of Gram-negative infection acquired during ICU stay.

Received 25 October 2020; Accepted 12 March 2021; Published 16 April 2021

Author affiliations: 'Department of Respiratory Medicine, Nottingham University Hospital NHS Trust, Nottingham NG5 1PB, UK; ${ }^{2}$ Division of Epidemiology and Public Health, School of Medicine, University of Nottingham, Clinical Sciences Building, Nottingham City Hospital Campus, Hucknall Road, Nottingham NG5 1PB, UK; ${ }^{3} \mathrm{NIHR}$ Nottingham Biomedical Research Centre, Queen's Medical Centre, Nottingham NG7 2UH, UK; 4 Division of Respiratory Medicine, School of Medicine, University of Nottingham, Queens Medical Centre, Derby Rd, Nottingham NG7 2UH, UK; ${ }^{5}$ University College London Hospitals NHS Foundation Trust, 250 Euston Rd, London NW1 2PG, UK; ' ${ }^{\circ}$ Salford Royal NHS Foundation Trust, Stott Ln, Salford M6 8HD, UK; ${ }^{7}$ Guy's and St Thomas' NHS Foundation Trust, Great Maze Pond, London SE1 9RT, UK; ${ }^{8}$ Antimicrobial Pharmacodynamics and Therapeutics, Department of Molecular and Clinical Pharmacology, University of Liverpool, Liverpool, L69 3GE, UK; ${ }^{9}$ Brighton and Sussex University Hospitals NHS trust, Eastern Road, Brighton BN2 1ES, UK; ${ }^{10}$ Newcastle Upon Tyne Hospitals NHS Foundation Trust, Freeman Rd, High Heaton, Newcastle upon Tyne NE7 7DN, UK; ${ }^{11}$ University Hospitals of Derby and Burton NHS Foundation Trust, Uttoxeter Road, Derby DE22 3NE, UK; ${ }^{12}$ Division of Infection, Immunity and Respiratory Medicine, NIHR Manchester Biomedical Research Centre, University of Manchester, Manchester, M23 9PT, UK; ${ }^{13}$ Department of Critical Care, Nottingham University Hospital NHS Trust, Nottingham NG5 1PB, UK; ${ }^{14}$ Division of Anaesthesia, School of Medicine, University of Nottingham, Queens Medical Centre, Derby Rd, Nottingham NG7 2UH, UK.

*Correspondence: Vadsala Baskaran, vadsala.baskaran1@nottingham.ac.uk

Keywords: COVID-19; co-infection; ICU; antibiotic resistance.

Abbreviations: APACHE, acute physiology and chronic health evaluation; BAL, bronchoalveolar lavage; BAME, Black, Asian and Minority Ethnicity;

CPAP, continuous positive airway pressure; ICNARC, Intensive Care National Audit and Research Centre; ICU, intensive care unit; LOS, length of stay;

$\mathrm{OR}$, odds ratio; RT-PCR, reverse transcriptase-polymerase chain reaction.

Supplementary material is available with the online version of this article.

001350 ๑ 2021 The Authors 


\section{INTRODUCTION}

During previous viral pandemics, reported co-infection rates and implicated pathogens have varied. In the 1918 influenza pandemic an estimated $95 \%$ of severe illness and death was complicated by bacterial co-infection, predominantly Streptococcus pneumoniae and Staphylococcus aureus [1].

As of 3 September 2020, over 25 million cases and 850,000 deaths due to COVID-19 infection have been reported worldwide [2]. The symptoms associated with COVID-19 infection are relatively non-specific. Fever and lower respiratory tract symptoms, such as a cough or breathlessness, are common in patients who require hospital care and radiological changes consistent with pneumonia are evident in up to $97 \%$ of these patients [3]. Confirmation of acute COVID-19 infection is reliant on a positive SARS-CoV-2 polymerase chain reaction (PCR) test result. The immune response to SARS-CoV2 infection includes a rise in IL- 6 and C-reactive protein (CRP), with higher levels associated with more severe disease $[4,5]$.

The contribution of secondary or co-pathogens to COVID-19 infection is not well understood. The lack of an effective antiviral agent against SARS-CoV2 combined with challenges in differentiating secondary bacterial co-infection from severe COVID-19 infection alone, has fostered the widespread use of empirical antibiotics in the immediate management of patients hospitalised with COVID-19 infection. Over the spring wave of the pandemic, $83.1 \%$ of hospitalised patients received empirical antibiotic treatment [6].

The utility of specific biomarkers such as procalcitonin to guide antibiotic therapy in severe respiratory tract infection, and specifically COVID-19 infection, is as yet uncertain $[7,8]$. In the meantime, a better understanding of the incidence of co-infection in patients with COVID-19 infection and the pathogens involved is necessary for effective antimicrobial stewardship. The primary objective of this study was to determine the rate of co-infection in critically ill adults with COVID-19 infection in England. Secondary aims were to describe the organisms, the characteristics of patients with co-infection and the antibiotic susceptibilities of identified bacteria.

\section{METHODS}

\section{Data source}

A retrospective observational multicentre study of co-infection in adults with confirmed COVID-19 requiring intensive care unit (ICU) admission was performed. Seven acute hospitals from across England participated in the study including large ( $>1,000$ beds) tertiary hospitals and medium (500-1,000 beds) district hospitals: Nottingham University Hospitals NHS Trust, Newcastle Upon Tyne Hospitals NHS Foundation Trust, Brighton and Sussex University Hospitals NHS Trust, Guy's and St Thomas' NHS Foundation Trust, Salford Royal NHS Foundation Trust, University Hospitals of Derby and Burton NHS Foundation Trust and University College London Hospitals NHS Foundation Trust.

\section{Study population}

Case inclusion criteria were adults aged $>16$ years with completed ICU admissions (discharged from or died whilst in ICU) for COVID-19 pneumonia (i.e. requiring Level 2 or Level 3 care according to the classification by the Intensive Care Society, UK) from disease emergence to 18 May 2020. SARS-CoV-2 was confirmed using reverse transcriptasepolymerase chain reaction (RT-PCR) from a respiratory specimen. Participating sites were asked to enter data for either: (1) all identified patients, or (2) a random selection of at least ten patients from across their eligible cohort. Where more than one critical care area existed at a participating site, a random selection from across areas was requested to avoid selection bias. Exclusion criteria were defined as: COVID-19 infection diagnosed $>48 \mathrm{~h}$ after hospital admission or a hospital admission in the last 14 days (hospital-acquired COVID-19) and patients transferred into ICU from a different hospital. Only the first admission to ICU was included.

\section{Data collection}

Personal information was removed at the point of participating site data entry onto a secure online database platform (REDCap Cloud). Data were gathered from electronic medical records. Fields collected were: demographics (age, gender, ethnicity, presence or absence of co-morbidity as defined in the Intensive Care National Audit and Research Centre (ICNARC) report on COVID-19 in critical care (Online Resource 1, available in the online version of this article) and type 2 diabetes mellitus); hospital admission details (date, days of symptom onset prior to admission and radiology findings); ICU details (date of admission, mechanical ventilation during the first $24 \mathrm{~h}$, advanced respiratory support (Online Resource 1), acute physiology and chronic health evaluation (APACHE II) score and outcomes); antibiotics received and all microbiology test results to the end of the ICU admission (including any identified antimicrobial resistance).

\section{Definitions}

Diagnostic microbiology tests were performed as per standard testing protocols within NHS laboratories at individual participating sites. Microbiology results included in the analysis were: standard culture (blood, sputum, trachealaspirate, bronchoalveolar lavage (BAL), urine) and validated culture-independent tests such as respiratory viral PCR (see Online Resource 2) and urinary antigens. Co-infection was defined as present if a likely pathogen was identified in a clinical sample taken for diagnostic purposes. Culture results were excluded if they were considered to represent contamination or colonisation. Specifically, this applied to the following situations: blood cultures yielding common skin contaminants in a single sample (coagulase-negative staphylococci, Micrococcus spp., viridans group streptococci, Propionibacterium spp., Corynebacterium spp., Bacillus spp.) without a concurrent positive culture from an indwelling line tip [9-11], Candida spp. cultured from respiratory and urinary catheter samples $[12,13]$, respiratory samples yielding Gram-positive organisms typically present in the 


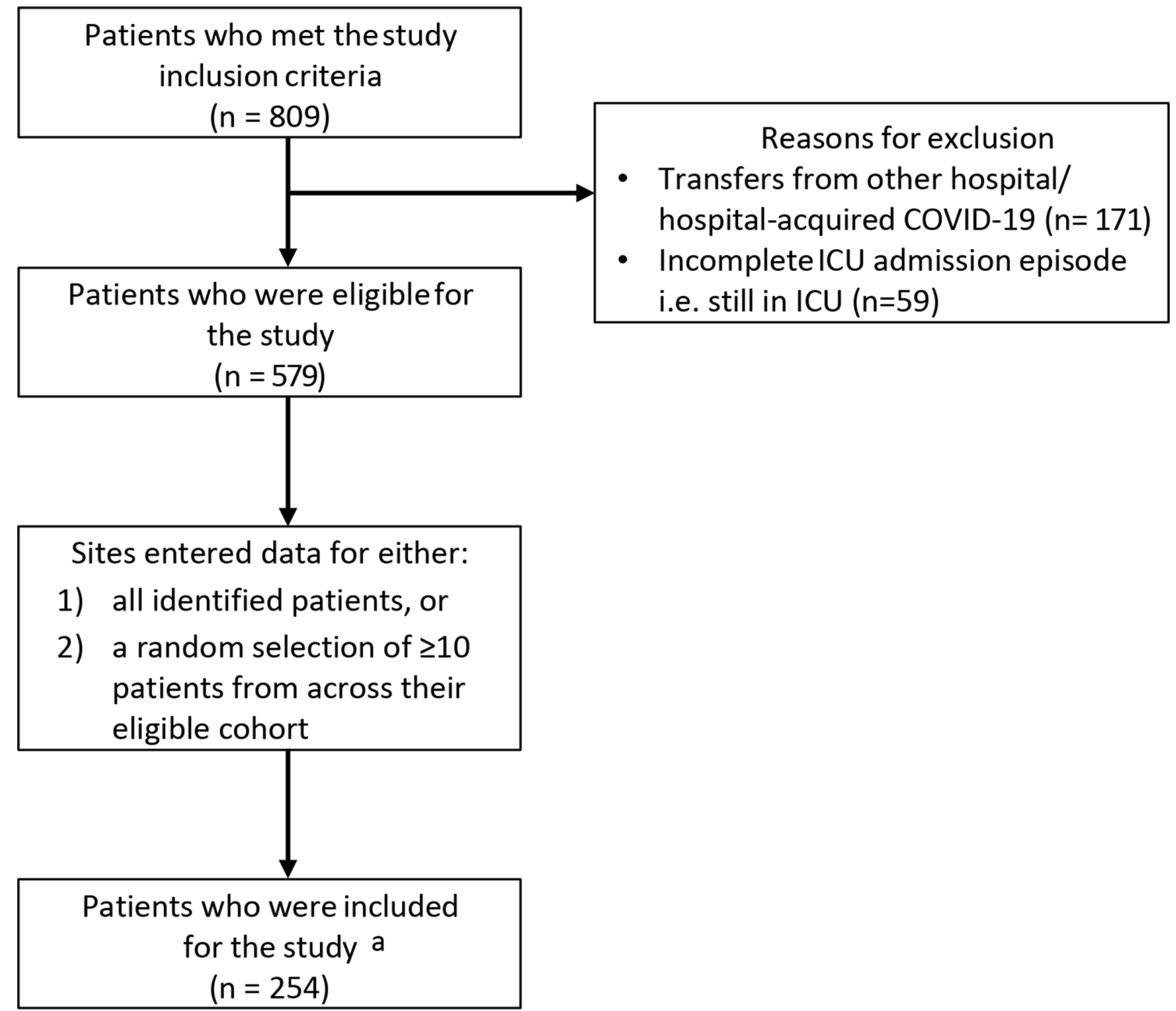

Fig. 1. Flowchart of study population. ${ }^{a}$ See Online Resource 3 in the supplementary material for exact breakdown.

oropharyngeal flora [14], growth of Enterococcus spp. in a single catheter urinary specimen [15]. Despite this effort, culture results from some non-sterile respiratory samples may represent colonisation, hence the term co-infection/ co-colonisation is used for respiratory samples. Radiology findings were defined based on the COVID-19 British Society of Thoracic Imaging reporting template [16]. Where both chest $\mathrm{CT}$ and CXR findings were available, chest $\mathrm{CT}$ findings were prioritised.

\section{Statistical analysis}

Demographics, clinical and disease characteristics were described using appropriate descriptive statistics for: (i) those with co-infection, and (ii) those without co-infection. Characteristics of patients in the study were also compared with the patients in the Intensive Care National Audit and Research Centre (ICNARC) report on COVID-19 in critical care, 22 May 2020. The proportion of co-infection (\%) was determined at three time points: on admission, within $48 \mathrm{~h}$, and during ICU admission (from day of ICU admission to ICU discharge or death in ICU). The co-infection rate was calculated per 1000 person-days based on the first co-infection detected in hospital per patient (person-time was determined from date of hospital admission to date of first co-infection, date of discharge from ICU or date of death in ICU, whichever came first for each patient). Univariate logistic regression analyses were conducted to determine the association between selected variables (age, gender, study site, ethnicity and co-morbidities) and the odds of (a) developing co-infection during admission, and (b) co-infection and mortality in ICU. Competing-risks regression analysis was conducted to assess if patients with co-infection had a longer length of hospital stay (from hospital admission to the end of ICU admission) than those without co-infection, with death as a competing-event. Co-pathogens were described separately for bacterial, viral and fungal infections. The proportion of bacterial co-pathogens with antimicrobial resistance was recorded.

An analysis of type of pathogens identified at different time points from admission was performed $(\leq 48 \mathrm{~h}$ and $>48 \mathrm{~h}$ following admission) to identify those with community 
Table 1. Characteristics of study population in comparison with ICNARC data

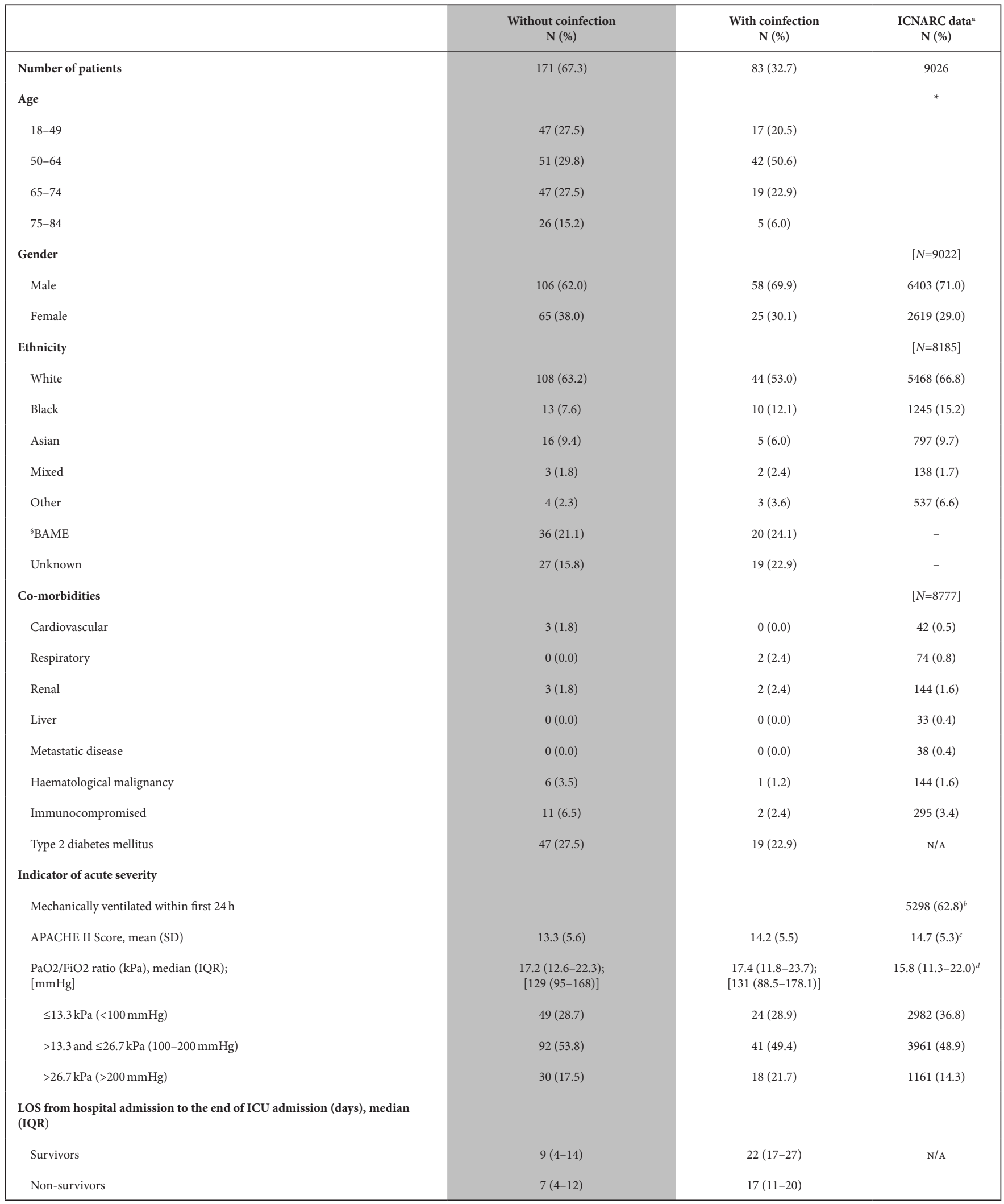

a. Intensive Care National Audit and Research Centre (ICNARC) report from 22 May 2020.

${ }^{*}$ Median age $=60(51-68)$.

Denominators: $b, \mathrm{~N}=8433, c, \mathrm{~N}=8648$ and $d, \mathrm{~N}=8104$

$\S$ BAME is the total of Black, Asian, Mixed andOther ethnicities 
Table 2. Organisms identified within $48 \mathrm{~h}$ of hospital admission

\begin{tabular}{|c|c|c|}
\hline Type of test & Potential pathogens & No of pathogens \\
\hline \multicolumn{3}{|l|}{$\begin{array}{l}\text { Tracheal aspirate or } \\
\text { sputum culture }\end{array}$} \\
\hline & Escherichia coli & 1 \\
\hline & Pseudomonas $\mathrm{sp}^{a}$ & 1 \\
\hline & Pseudomonas aeruginosa & 1 \\
\hline & $\begin{array}{l}\text { Enterobacter cloacae complex } \\
\qquad(A m p C)\end{array}$ & 1 \\
\hline & $\begin{array}{l}\text { Staphylococcus aureus } \\
(\mathrm{MSSA})^{a}\end{array}$ & 2 \\
\hline \multicolumn{3}{|l|}{ BAL PCR/ culture } \\
\hline & $\begin{array}{c}\text { Staphylococcus aureus (MSSA } \\
\text { and MRSA) }\end{array}$ & 2 \\
\hline & Klebsiella pneumoniae & 1 \\
\hline \multicolumn{3}{|l|}{ Other tests } \\
\hline $\begin{array}{l}\text { Pneumococcal } \\
\text { urinary antigen test }\end{array}$ & Streptococcus pneumoniae ${ }^{a}$ & 2 \\
\hline MSU & Escherichia coli & 2 \\
\hline \multirow[t]{2}{*}{ RT-PCR } & Mycoplasma pneumoniae & 1 \\
\hline & Metapneumovirus ${ }^{a}$ & 1 \\
\hline \multicolumn{3}{|c|}{$\begin{array}{l}\text { a. Pathogens identified on the day of admission (Pseudomonas sp, } \\
\text { one out of two MSSA and one out of two S. pneumoniae identified, } \\
\text { and Metapneumovirus), total=4. } \\
\text { b, One out of two organisms was Methicillin-resistant } \\
\text { Staphylococcus aureus (MRSA). The same patient also had MRSA in } \\
\text { pleural fluid culture after } 48 \mathrm{~h} \text { into hospital admission. }\end{array}$} \\
\hline
\end{tabular}

vs hospital-acquired co-infection. Pathogens identified within $48 \mathrm{~h}$ of hospital admission were listed by type of test performed. A sub-analysis of the hospital-acquired co-infection was performed to identify the type of pathogens detected early (3-7 days into hospital admission) and late ( $>7$ days into hospital admission). Statistical analyses were performed using Stata MP/15.1.

\section{RESULTS}

Of 579 eligible patients during the study period, 254 patients with completed ICU episodes were studied (Fig. 1).

The median age of the study cohort was 59 years (IQR 49-69, range 19-84) and 164 (64.6\%) patients were male; similar to corresponding data from the ICNARC cohort (Table 1) [17]. Patients were admitted to hospital between 21 Feb 2020 and 1 May 2020. The median time from onset of symptoms to admission was 7 days (IQR 5-10). The median time from hospital admission to ICU admission was 1 day (IQR 0-2). Antibiotics were prescribed to $35(13.8 \%)$ patients before hospital admission and to 228 (89.8\%) patients within $48 \mathrm{~h}$ of admission. Throughout the course of admission, 241 (94.9\%) of patients received antibiotics at some point.

The overall median length of stay (LOS) in ICU was 9 days (IQR 4-17); 10 days (IQR 4-18) for survivors and 9 days (IQR 5-15) for non-survivors. One hundred and fifty-one patients (59.5\%) were mechanically ventilated within $24 \mathrm{~h}$ of admission, and 158 patients (62.2\%) received advanced respiratory support (invasive ventilation, CPAP via trans-laryngeal tube, extracorporeal respiratory support) during admission. Of those who were discharged from ICU ( $n=172$ patients), two patients $(1.2 \%)$ died in hospital, 147 patients $(85.5 \%)$ were discharged from hospital and 23 patients $(13.4 \%)$ remained in hospital at the end of the study.

All patients had either a CXR ( $n=246$ patients) and/or a chest CT scan ( $n=74$ patients). Classic/ probable COVID-19 radiographic changes were recorded in 209 patients $(82.3 \%)$, five $(2 \%)$ had normal imaging, $27(10.6 \%)$ had indeterminate changes and 13 (5.1\%) had non-COVID19 findings.

In total, co-infection/ co-colonisation was identified in 83 $(32.7 \%)$ patients from hospital admission to the end of ICU stay; median time to co-infection/ co-colonisation was 9 days (IQR 6-14). The list of identified potential pathogens and contaminants from standard cultures (blood, BAL, sputum and tracheal aspirate) is available in Online Resource 4. On the day of admission, potential co-pathogens were identified in four patients (1.6\%), rising to $14(5.5 \%)$ patients within the first $48 \mathrm{~h}$ of hospital admission. Fifteen potential pathogens were identified from 14 patients within $48 \mathrm{~h} ; 14$ bacterial and one viral pathogen (Table 2). None of these potential pathogens were identified from blood culture. In a sensitivity analysis excluding the hospital which contributed a third of cases, the $48 \mathrm{~h}$ co-infection/ co-colonisation rate remained similar (Online Resource 5). The commonest potential co-pathogen within $48 \mathrm{~h}$ of hospital admission was $S$. aureus, three methicillin-susceptible (MSSA) and one methicillin-resistant S. aureus (MRSA) (four patients). Two positive Mycoplasma IgG/ IgM tests in separate patients were deemed false positives and excluded from the analysis. The number of tests performed within $48 \mathrm{~h}$ of hospital admission are listed in Online Resource 6, by type of tests and study site. For bacterial co-pathogens, the antimicrobial susceptibilities are described in Online Resource 7 in the supplementary material.

Beyond $48 \mathrm{~h}$ of hospital admission to the end of ICU stay, 124 potential co-pathogens were identified in 77 (30.3\%) patients; 29 potential pathogens from days $3-7$ and 95 potential pathogens from day 8 onwards (Fig. 2). The co-infection/ co-colonisation rate $>48$ hours after admission was 27.0 per 1000 person-days (95\% CI 21.3-34.1). All were bacterial pathogens $(n=122)$ except for two fungal organisms. The commonest potential co-pathogens identified were Gramnegative bacteria, including Klebsiella spp. (23 patients) and Escherichia coli (20 patients). No viral co-pathogens were detected. Of the two fungal co-pathogens, one was Aspergillus fumigatus from a tracheal aspirate culture obtained on day 5 in a 54 year old male. The other was Candida parapsilosis from 


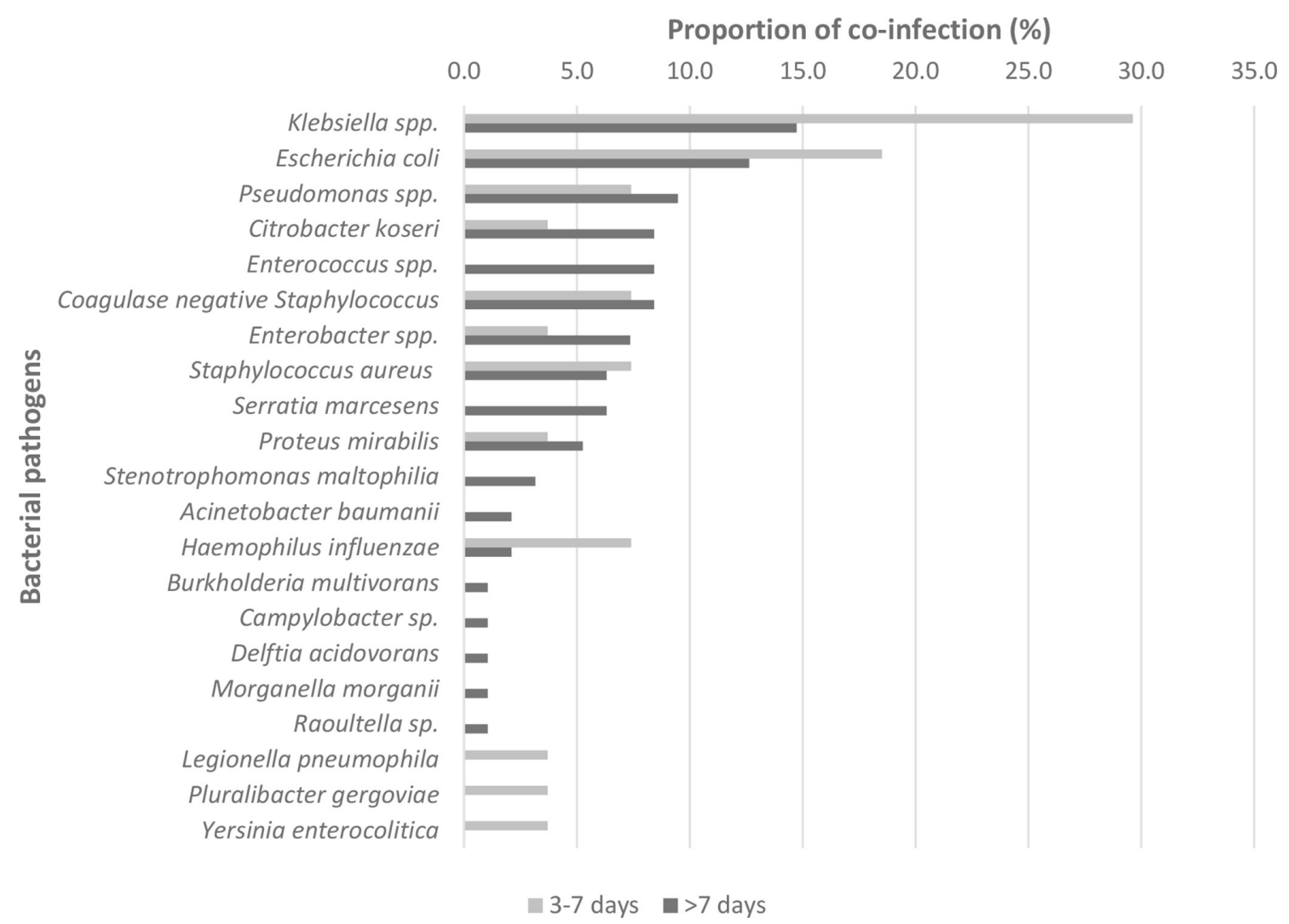

Fig. 2. Bacterial pathogens detected after $48 \mathrm{~h}$ of hospital admission; 124 potential pathogens detected. Reported as proportion (\%) of the total number of bacterial pathogens detected within '3-7 days' and '> 7 days' from hospital admission.

a blood culture taken at day 7 in a 55 year old lady. Neither patient had any pre-existing co-morbidities.

On univariate analyses, patients aged 50-64 years were more likely to have a co-infection/ co-colonisation than those aged 18-49 years. No other significant association was found (Table 3). Patients with co-infections/ co-colonisation were more likely to die in ICU (with coinfections/ co-colonisation, $n=34$ vs without coinfections/ co-colonisation, $n=48$, crude OR $1.78,95 \% \mathrm{CI} 1.03-3.08, P=0.04)$ and had a longer hospital LOS (measured from admission to hospital to the end of ICU admission, subhazard ratio (likelihood of discharge from ICU $)=0.53,95 \%$ CI $0.39-0.71, P<0.001)$.

\section{DISCUSSION \\ Principal findings}

Bacterial co-infection/ co-colonisation within $48 \mathrm{~h}$ of hospital admission for COVID-19 infection in adults was uncommon; $1.6 \%$ on admission and $5.5 \%$ within $48 \mathrm{~h}$. The commonest pathogens identified within the first $48 \mathrm{~h}$ of hospital admission were Staphylococcus aureus and Streptococcus pneumoniae. The proportion of pathogens detected increased with duration of ICU stay and consisted largely of Gram-negative bacteria, particularly Klebsiella pneumoniae and Escherichia coli. The co-infection/ co-colonisation rate $>48 \mathrm{~h}$ after admission was 27.0 per 1000 person-days (95\% CI 21.3-34.1).

\section{Comparison with literature}

Concern regarding co-infection during viral pandemics, specifically respiratory co-infection with a bacterial pathogen, is borne from previous experience in influenza. During the 2009 H1N1 influenza A pandemic, early co-infection/ co-colonisation rates were high; $22.5 \%$ within $72 \mathrm{~h}$ of admission in adults requiring critical care [18]. In contrast, limited evidence from studies of Severe Acute Respiratory Syndrome and Middle East Respiratory Syndrome suggest lower co-infection rates (10.3-18.5\%) [19, 20]. In COVID19 , systematic reviews based on studies predominantly from China reported low estimates $(<7 \%)$ of bacterial co-infection [21-23]. In the UK, retrospective single-centre studies have observed low rates of bacterial co-infection [24-26]. Hughes et al. detected early bacterial infection ( $0-5$ days from admission) in $3.2 \%$ of all hospitalised patients $(13.5 \%$ of those requiring critical care), increasing to $6.1 \%$ throughout admission [25]. Youngs et al. reported bacterial co-infection within $48 \mathrm{~h}$ of admission to ICU in $8 \%$ of patients with COVID-19 compared to $58 \%$ of patients with influenza, with no difference in the incidence of late infection between the two groups [26]. In the US, higher early bacterial co-infection rates (16.6\%) 
Table 3. Univariate logistic regression analyses investigating the association between variables of interest and odds of developing coinfection/ co-colonisation

\begin{tabular}{|c|c|c|c|}
\hline & \multicolumn{2}{|c|}{ Crude OR (95\% CI) } & $p$ value \\
\hline \multicolumn{4}{|l|}{ Number of patients } \\
\hline \multicolumn{4}{|l|}{ Age } \\
\hline $18-49$ & \multicolumn{2}{|c|}{1 (Reference) } & \\
\hline $50-64$ & 2.28 & $(1.14-4.53)$ & $0.019^{*}$ \\
\hline $65-74$ & 1.12 & $(0.52-2.41)$ & 0.777 \\
\hline $75-84$ & 0.53 & $(0.18-1.61)$ & 0.263 \\
\hline \multicolumn{4}{|l|}{ Gender } \\
\hline Male & \multicolumn{2}{|c|}{1 (Reference) } & \\
\hline Female & 0.70 & $(0.40-1.23)$ & 0.218 \\
\hline \multicolumn{4}{|l|}{ Ethnicity } \\
\hline White & \multicolumn{2}{|c|}{1 (Reference) } & \\
\hline Black & 1.89 & $(0.77-4.62)$ & 0.164 \\
\hline Asian & 0.77 & $(0.26-2.22)$ & 0.625 \\
\hline Mixed & 1.64 & $(0.26-10.13)$ & 0.597 \\
\hline Other & 1.84 & $(0.40-8.57)$ & 0.437 \\
\hline $\mathrm{BAME} \dagger$ & 1.36 & $(0.71-2.61)$ & 0.349 \\
\hline Unknown & 1.73 & $(0.87-3.42)$ & 0.117 \\
\hline \multicolumn{4}{|l|}{ Co-morbidities } \\
\hline Cardiovascular & - & & \\
\hline Respiratory & - & & \\
\hline Renal & 1.38 & $(0.23-8.43)$ & 0.725 \\
\hline Liver & - & & \\
\hline Metastatic disease & - & & \\
\hline Haematological malignancy & 0.34 & $(0.04-2.83)$ & 0.316 \\
\hline Immunocompromise & 0.36 & $(0.08-1.65)$ & 0.187 \\
\hline Type 2 diabetes mellitus & 0.78 & $(0.42-1.44)$ & 0.434 \\
\hline
\end{tabular}

* $p$ value of $<0.05$ denotes a significant difference " median and IQR. †BAME is the total of Black, Asian, Mixed and Other ethnicities.

were identified by Crotty et al.; respiratory cultures positive for oral bacteria flora constituted 15/25 of these cases [27]. In contrast to studies that relied on predominantly culturebased techniques, Kreitmann et al. identified early bacterial co-infection in $27.7 \%$ (13/47) of their prospective cohort of ventilated patients using a multiplex PCR assay with only one case identified by conventional culture [28]. In France, a single centre study using three multiplex PCR assays performed on respiratory specimens or nasopharyngeal swabs in addition to standard culture techniques retrospectively identified bacterial co-infection in $28 \%$ of 92 ICU admissions [29]. Variations in case definitions, diagnostic testing and geography may partly account for the differences observed between studies although overall, there is a suggestion that increased severity of disease, particularly when ICU care is required, is associated with increased rates of co-infection.

The prevalence of nosocomial infection is $20.6 \%$ and increases with duration of ICU stay [30,31]. Our observed co-infection/ co-colonisation rate is relatively high, consistent with a patient cohort with long ICU stays (median 10 days) and requiring high levels of respiratory support.

Consistent with reports from other studies, the commonest potential co-infecting bacteria identified within $48 \mathrm{~h}$ of admission was $S$. aureus $[25,28,32]$. In patients in whom early co-infection is suspected clinically, due consideration of $S$. aureus is warranted. However, the rate of $S$. aureus co-infection is markedly lower than that observed in pandemic influenza, suggesting it is a less significant issue with COVID-19 infection [18]. The predominant late pathogens observed were Gram-negative bacteria, particularly K. pneumoniae. These pathogens are commonly associated with hospital and ventilator-acquired pneumonia and have been reported as common co-pathogens in COVID-19 infections, particularly ICU cohorts [21, 22, 33-35]. The predominance of Gram-negative bacteria in these studies likely reflects nosocomial infection following prolonged ICU stay and empirical antibiotic use.

Viral co-pathogen was identified in one patient in our cohort; lower than the $3 \%$ (95\% CI 1-6\%) viral co-infection rate reported in systematic reviews and in contrast to the $20.7 \%$ viral co-detection rate reported by Kim et al. in Northern California [21,36]. The 2019/20 influenza season in the UK ended in late March [37]. Other UK cohorts recruited during the spring wave of COVID-19 (March - May 2020) similarly reported very little or no viral co-infection $[25,35]$.

\section{Strengths and limitations}

This pragmatic multicentre study provides novel data on both community-acquired and nosocomial co-infection/ co-colonisation in patients with COVID-19 requiring ICU care in England. The ICU cohort represents those with severe disease who were subject to more rigorous microbiology sampling. A key limitation of the study is its retrospective observational design subject specifically to case selection, ascertainment and sampling biases. Inclusion of consecutive eligible patients was not feasible due to pandemic workload constraints. To minimise case selection bias, participating sites submitted a random sample of their eligible cohort, although random sampling methods were not standardised. The impact of ascertainment bias due to differences in the proportion of eligible cases submitted by each institution was reduced through the participation of multiple centres. The study cohort was comparable to the ICNARC cohort except for an under-representation of patients of Black, Asian and Minority Ethnicity (BAME). Our results may not be applicable to settings with larger BAME populations. Restriction of our cohort to those with completed ICU admissions excluded: (i) frailer patients in whom ICU care was deemed 
not appropriate, and (ii) patients with very long ICU stays. Co-infection, particularly nosocomial infection, may be higher in these patients.

A second key limitation is that although results likely to represent contamination were excluded, some pathogens found in respiratory tract samples may represent colonisation rather than active co-infection. However, as sputum samples sent from ICU should reflect clinical concern of lower respiratory tract infection (especially during the pandemic timeframe) and positive culture represents predominant presence of a pathogen rather than as part of mixed flora, we have taken these results to represent infection. If colonising pathogens were wrongly attributed as causing infection, the direction of bias would be towards falsely higher co-infection rates observed in our study.

Thirdly, reliance on culture dependent techniques may have falsely decreased co-infection rates. Antibiotic use prior to admission was low (13.8\%), increasing the reliability of culture-based methods on admission. However, detection of pathogens later into admission would have been influenced by sampling bias and the use of empirical antibiotics. Fourthly, although seven hospitals participated in this study, one study site contributed a third of cases; observed $48 \mathrm{~h}$ co-infection/ co-colonisation rate excluding this site was, however, similar to overall results.

\section{Implications for future work}

Notwithstanding these limitations, our data indicate that early in hospitalisation, bacterial co-infection in COVID-19 is very uncommon and support the recommendations that empirical antibiotics should not be started routinely in primary care or at the point of hospital admission without clinical suspicion of bacterial infection [8]. The high rate of co-infection found late in illness among patients requiring ICU and involving nosocomial pathogens is concerning. It is plausible that reducing unnecessary early antibiotic exposure in patients with COVID-19 could reduce their risk of late, Gram-negative, potentially antibiotic resistant infections $[38,39]$.

Since study completion, dexamethasone has been shown to decrease mortality in patients hospitalised with COVID-19 who require oxygen support or invasive mechanical ventilation [40]. Consequently, dexamethasone has become established as standard of care for these patients in many countries. This may increase the already high rate of bacterial co-infection we observed in ICU-treated patients. A high level of microbiological vigilance is recommended as part of the management of these patients. In the setting of seasonal changes in respiratory pathogens, ongoing surveillance for co-infections in patients hospitalised with COVID-19, ideally through prospective studies with standardised sampling protocols, is advised.

\section{Availability of data and material}

Data are available from the corresponding author on reasonable request.
Funding information

This research was funded by the NIHR Nottingham Biomedical Research Centre. The views expressed are those of the author(s). The funders had no role in the design, analysis or write up of this manuscript. Grant number: BRC-1215-20003.

Acknowledgement

We wish to thank Mr Glenn Hearson for building the study database on the secure online database platform (REDCAP Cloud).

\section{Author contributions}

All included authors fulfil the criteria of authorship; V. B. and H. L., are joint first authors for this manuscript. W. S. L., V. B. and H. L., had substantial contributions to the study conception and design. All authors had substantial contributions to the data acquisition. V. B., performed the analyses. All authors had substantial contributions to the results interpretation. VB and HL wrote the original draft. All authors revised the manuscript critically for important intellectual content, provided the final approval of the version to be published and agreed to be accountable for all aspects of the work in ensuring that questions related to the accuracy or integrity of any part of the work are appropriately investigated and resolved.

\section{Conflicts of interest}

Professor Lim reports grants from National Institute for Health Research (NIHR), grants from Pfizer, outside the submitted work. Paul Dark is funded by NIHR Manchester BRC as sub-theme lead in Respiratory Infections.

Ethical statement

Ethical approval was given by HRA and REC; protocol number:20RM040, IRAS project ID:284341. Section 251 support from the Confidentiality Advisory Committee for use of anonymised NHS patient data was not required according to the temporary General Notice issued for COVID-19 purposes by the Secretary of State for Health and Social Care under the Health Service Control of Patient Information Regulations 2002.

References

1. McCullers JA. The co-pathogenesis of influenza viruses with bacteria in the lung. Nat Rev Microbiol 2014:12:252-262.

2. WHO. WHO Covid-19 Dashboard 2020 [02/06/20]. Available from. https://covid19. who.int/?gclid=CjwKCAjw8df2BRA3EiwAvfZW aJWnmCWZBUjJdJZGVdH4hGENu8orjqQTHDsIst5u_gYXoQcl8sS_ ZxoClxEQAvD_BwE

3. Guan W-jie, Ni Z-yi, Hu Y, Liang W-hua, Ou C-quan et al. Clinical characteristics of coronavirus disease 2019 in China. N Engl J Med Overseas Ed 2020;382:1708-1720.

4. Zhou F, Yu T, Du R, Fan G, Liu Y et al. Clinical course and risk factors for mortality of adult inpatients with COVID-19 in Wuhan, China: a retrospective cohort study. Lancet 2020:395:1054-1062.

5. Herold T, Jurinovic V, Arnreich C, Lipworth BJ, Hellmuth JC et al. Elevated levels of IL-6 and CRP predict the need for mechanical ventilation in COVID-19. J Allergy Clin Immunol 2020;146:128-136.

6. ISARIC ISARaeIC. Clinical data report 2020.

7. Tan C, Huang Y, Shi F, Tan K, Ma Q. C-Reactive protein correlates with CT findings and predicts severe COVID-19 early. J Med Virol.

8. National Institute for Health and Care Excellence N. COVID-19 rapid guideline: antibiotics for pneumonia in adults in hospital:NICE; 2020 [cited 2020 June 2020]. Available from. https://www.nice.org.uk/guidance/ ng173/chapter/3-Initial-approach-to-antibiotic-treatment-choices

9. Bekeris LG, Tworek JA, Walsh MK, Valenstein PN. Trends in blood culture contamination: a College of American pathologists Q-Tracks study of 356 institutions. Arch Pathol Lab Med 2005;129:1222-1225.

10. Hall KK, Lyman JA. Updated review of blood culture contamination. Clin Microbiol Rev 2006;19:788-802.

11. Freeman JT, Chen LF, Sexton DJ, Anderson DJ. Blood culture contamination with enterococci and skin organisms: implications for surveillance definitions of primary bloodstream infections. Am $J$ Infect Control 2011:39:436-438.

12. Gajdács M, Dóczi I, Ábrók M, Lázár A, Burián K. Epidemiology of candiduria and Candida urinary tract infections in inpatients and 
outpatients: results from a 10-year retrospective survey. Cent European J Urol 2019;72:209-214.

13. Pendleton KM, Huffnagle GB, Dickson RP. The significance of Candida in the human respiratory tract: our evolving understanding. Pathog Dis 2017:75 [Epub ahead of print 01 Apr 2017].

14. Public Health England P. UK SMI B 57 issue 3.5 (May 2019): investigation of bronchoalveolar lavage, sputum and associated specimens May 2019 02/06/2020; (issue 3.5). Available from. https://www.gov.uk/government/publications/smi-b-57-investigation-of-bronchoalveolar-lavage-sputum-and-associatedspecimens

15. Lin E, Bhusal Y, Horwitz D, Shelburne SA, Trautner BW. Overtreatment of enterococcal bacteriuria. Arch Intern Med 2012;172:33-38.

16. Britiish Sociecty of Thoracic Imaging B. Covid-19 BSTI reporting templates and codes 2020 [updated 22/05/202002/06/2020]. Available from. https://www.bsti.org.uk/covid-19-resources/ covid-19-bsti-reporting-templates/

17. Intensive Care National Audit and Research Centre I. Online reports 2020 [June 2020]. Available from. https://onlinereports. icnarc.org/Home

18. Rice TW, Rubinson L, Uyeki TM, Vaughn FL, John BB et al. Critical illness from 2009 pandemic influenza A virus and bacterial coinfection in the United States. Crit Care Med 2012;40:1487-1498.

19. Arabi YM, Al-Omari A, Mandourah Y, Al-Hameed F, Sindi AA et al. Critically ill patients with the middle East respiratory syndrome: a multicenter retrospective cohort study. Crit Care Med 2017:45:1683-1695

20. Jang T-N, Yeh DY, Shen S-H, Huang C-H, Jiang J-S et al. Severe acute respiratory syndrome in Taiwan: analysis of epidemiological characteristics in 29 cases. J Infect 2004;48:23-31.

21. Lansbury L, Lim B, Baskaran V, Lim WS. Co-Infections in people with COVID-19: a systematic review and meta-analysis. J Infect.

22. Langford BJ, So M, Raybardhan S, Leung V, Westwood D et al. Bacterial co-infection and secondary infection in patients with COVID-19: a living rapid review and meta-analysis. Clin Microbiol Infect 2020;26:1622-1629.

23. Rawson TM, Moore LSP, Zhu N, Ranganathan N, Skolimowska K et al. Bacterial and fungal coinfection in individuals with coronavirus: a rapid review to support COVID-19 antimicrobial prescribing. Clin Infect Dis 2020;71:2459-2468.

24. Adler H, Ball R, Fisher M, Mortimer K, Vardhan MS. Low rate of bacterial co-infection in patients with COVID-19. Lancet Microbe 2020;1:e62.

25. Hughes S, Troise O, Donaldson H, Mughal N, Moore LSP. Bacterial and fungal coinfection among hospitalized patients with COVID-19: a retrospective cohort study in a UK secondary-care setting. Clin Microbiol Infect 2020;26:1395-1399.
26. Youngs J, Wyncoll D, Hopkins P, Arnold A, Ball J et al. Improving antibiotic stewardship in COVID-19: bacterial co-infection is less common than with influenza. J Infect 2020;81:e55-e57.

27. Crotty MP, Akins RL, Nguyen AT, Slika R, Rahmanzadeh K. Investigation of subsequent and co-infections associated with SARS-CoV-2 (COVID19) in hospitalized patients. medRxiv 2020;2020.05.29.20117176.

28. Kreitmann L, Monard C, Dauwalder O, Simon M, Argaud L. Early bacterial co-infection in ARDS related to COVID-19. Intensive Care Med 2020;46:1787-1789.

29. Contou D, Claudinon A, Pajot $\mathrm{O}$, Micaëlo M, Longuet Flandre $P$ et al. Bacterial and viral co-infections in patients with severe SARSCoV-2 pneumonia admitted to a French ICU. Ann Intensive Care 2020;10:119.

30. Vincent J-L. Nosocomial infections in adult intensive-care units The Lancet 2003:361:2068-2077.

31. Vincent J-L, Rello J, Marshall J, Silva E, Anzueto A et al. International study of the prevalence and outcomes of infection in intensive care units. JAMA 2009;302:2323-2329.

32. Nori $P$, Cowman $K$, Chen V, Bartash R, Szymczak W et al. Bacterial and fungal coinfections in COVID-19 patients hospitalized during the new York City pandemic surge. Infect Control Hosp Epidemiol 2021:42:1-13

33. Jones RN. Microbial etiologies of hospital-acquired bacterial pneumonia and ventilator-associated bacterial pneumonia. Clin Infect Dis 2010;51 Suppl 1:S81-S87.

34. Garcia-Vidal C, Sanjuan G, Moreno-García E, Puerta-Alcalde P Garcia-Pouton N. Incidence of co-infections and superinfections in hospitalised patients with COVID-19: a retrospective cohort study. Clin Microbiol Infect.

35. Dhesi Z, Enne VI BD, Livermore DM, High J, Russell C. Organisms causing secondary pneumonias in COVID-19 patients at 5 UK ICUs as detected with the FilmArray test. medRxiv 2020;2020.06.22.20131573.

36. Kim D, Quinn J, Pinsky B, Shah NH, Brown I. Rates of co-infection between SARS-CoV-2 and other respiratory pathogens. JAMA 2020;323:2085.

37. Public Health England P. PHE National Influenza Report - Week 32 report. Report. 2020 06/08/2020. Report No.: Week 32.

38. Chastre J, Wolff M, Fagon J-Y, Chevret S, Thomas F et al. Comparison of 8 vs 15 days of antibiotic therapy for ventilator-associated pneumonia in adults: a randomized trial. JAMA 2003;290:2588-2598.

39. Singh N, Rogers P, Atwood CW, Wagener MM, Yu VL. Short-course empiric antibiotic therapy for patients with pulmonary infiltrates in the intensive care unit. A proposed solution for indiscriminate antibiotic prescription. Am J Respir Crit Care Med 2000;162:505-511.

40. Dexamethasone in Hospitalized Patients with Covid-19. Preliminary report. N Engl J Med 2020.

\section{Five reasons to publish your next article with a Microbiology Society journal}

1. The Microbiology Society is a not-for-profit organization.

2. We offer fast and rigorous peer review - average time to first decision is 4-6 weeks.

3. Our journals have a global readership with subscriptions held in research institutions around the world.

4. $80 \%$ of our authors rate our submission process as 'excellent' or 'very good'.

5. Your article will be published on an interactive journal platform with advanced metrics.

Find out more and submit your article at microbiologyresearch.org. 